\title{
Radiation Therapy for Prostate Cancer Not Operated
}

\section{Nkoua Epala B*, Vestris P, Bougas S and Vinh-Hung V}

Clarac Hospital, Martinique University Hospital Center (CHUM), Brazzaville, Congo

*Corresponding Author: Nkoua Epala B, Clarac Hospital, Martinique University

Hospital Center (CHUM), Brazzaville, Congo.
Received: May 13, 2021

Published: June 05, 2021

(C) All rights are reserved by Nkoua Epala B., et al.

\begin{abstract}
Prostate cancer is the most common male cancer in Martinique. Radiation therapy plays a key role in the management of prostate cancer at the localized stage, in particular the intermediate and high-risk risks according to D'Amico's classification, but also at the locally advanced stage. The patients selected in this study all benefited from conformal radiotherapy with Volumetric Intensity Modulation by Arc Therapy in the acronym VMAT. It seemed appropriate to report our findings on the treatment of prostate cancer by VMAT at the Clarac Hospital of the University Hospital Center (CHU) in Martinique.
\end{abstract}

Keywords: Cancer Prostate; Localized; Locally Advanced; Radiotherapy

\section{Introduction}

Prostate cancer is the most common male cancer in Martinique according to the general register of cancers of Martinique. Radiotherapy Apia plays a key role in the management of prostate cancer at the localized stage, including intermediate and high-risk risks according to the classification of D'Amico but also at the locally advanced stage [1]. Among the new techniques currently used in the prostate cancer priis the Irradiation with Modulation of Volume Intensity by Arc Therapy in the acronym VMAT made possible in Martinique thanks to a device called Novalis Tx or TrueBEAM. The "VMAT" technique provides better local control, good coverage of different target volumes and maximum protection of risky or healthy gold games [2]. We thought it was appropriate to report our findings on the treatment of prostate cancer by VMAT at the Clarac Hospital of the University Hospital Center (CHU) in Martinique.

\section{Patients and Methods}

We undertook a descriptive retrospective study in the radiotherapy department of the Clarac Hospital of the Hospital A Hospital One Iversitaire (CHU) of Martinique over a period of 12 months all ant from 01 January 2019 to 31 December 2019. To be selected in our study, the files should include: - A diagnostic check-up containing a pelvic MRI and PSA levels; A histological confirmation of prostate cancer by biopsy samples; $\mathrm{U} n$ distance extension report includes a thoraco-abdomino pelvien scanner with or without bone scans and/or a PET scan at 18F-Choline depending on the stage at diagnosis.

Un pre-therapeutic assessment including a hemogram, a kidney and liver check-up in order to explore vital functions before the start of radiotherapy. A certainty that the patients have not had surgery (no prostatectomy).

The patients had referred to the radiotherapy department by the urology department of the M Artinique University Hospital and 
some private clinics in the area. All files were spent in a multidisciplinary consultation meeting (PCR) and decisions made collegially at the so-called CPR were recorded in the patients' files.

A prelude to the treatment, the simulation, during which the patients benefited from a dosimetric scanner thanks to an apply called simulator scanner allowed the acquisition of the images. Images acquired during the dosimetric scanner were transferred to a bypass station for target volumes and organs at risk. The fusion of the images of the anterior pelvic MRI with those of the scanner allowed a good delineation. After the delineation of the different target volumes and organs at risk as well as the prescription of doses at different volumes by the radio doctor, the turn was up to the dosimetrist to achieve dosimetry. Next, the medical physicist should verify and validate the calculation and homogeneous distribution of the prescribed doses to the different target volumes and ensure the maximum protection of the organs at risk.

The work of the radiotherapist duo and medical physicist was subsequently presented to the internal radiotherapy staff for validation of the proposed protocol. Thus, after the analysis of the Dose Volume Histogram (HDV), which is the statistical summary of dosimetry to the staff, the therapeutic protocol was validated and the patients programmed to begin treatment. The stress of doses to organs at risk must be respected, including bladder, rectum and intestinal handles. Thus the following constraints were met: -vessie V65 -lt; $50 \%$ (50\% of the prescribed dose must not exceed 65 Grays). -rectum V 50-lt; 50\% (50\% of the prescribed dose should not exceed 50 Grays). -small intestine V50 'lt; $35 \mathrm{~cm}^{3}$ and V40 'lt; 200 $\mathrm{cm}^{3}$ All patients in our series have been irradiated by the "VMAT" technique. The "VMAT" technique synthesizes the latest technical developments in radiotherapy and combines image-guided conformational radiotherapy with intensity modulation. It is an Irradiation with Volumetric Intensity Modulation by Arc Therapy that offers the possibility of irradiating the cancerous tissues with more precision, thanks to a modulation of the beams of irradiation on a complete arc of 360 degrees. Unlike tomotherapy, which is also a method of conformational irradiation with modulation of intensity by circular action, the VMAT offers a faster treatment $115 \mathrm{~min}$ of treatment) and which acts on reduced areas of intensity. The Novalis Tx or TrueBEAM device from the Clarac Hospital of the Hospital of Martinique makes possible this technique "VMAT" thus allows so much better local control, good coverage of the different target volumes and maximum protection of organs at risk. In our series, all patients received the dose of 76 grays of 2 grays per fraction on the entire prostate and 53.2 grays of 1.4 grays per fraction in simultaneous integrated boost (SIB) on the pelvis with or without seminal vesicles in 38 sessions at a rate of 5 sessions per week for 7 weeks. Depending on the stage of diagnosis and in accordance with the classification of D'Amico (low risk, intermediate risk and high risk), intermediate-risk patients had benefited from the combination of radiation and short-term hormone therapy (6 months) while patients at high risk and in locally advanced stages had benefited from the combination of radiotherapy and hormone therapy for the long term (3years). Low-risk patients were not part of our series (active surveillance or first surgery). During treatment, weekly monitoring of patients is mandatory.

\section{Results}

During the 12-month study period, 192 files of patients treated with radiation therapy for non-operated prostate cancer that met the inclusion criteria of our study were collected.

The average age is 71 years for extremes ranging from 54 to 93 years.

The average referral time for patients in radiation therapy was 2 months.

The histological type was essentially adenocarcinoma. The patients were classified as salton the classification of DAmico.

\begin{tabular}{|l|c|c|c|c|}
\hline $\begin{array}{c}\text { Age ranges } \\
\text { (years) }\end{array}$ & $\begin{array}{c}\text { Intermediate } \\
\text { Risk }\end{array}$ & $\begin{array}{c}\text { High } \\
\text { Risk }\end{array}$ & $\begin{array}{c}\text { Locally } \\
\text { Advanced } \\
\text { Risk }\end{array}$ & Total \\
\hline $50-60$ & 5 & 4 & 9 & 18 \\
$61-70$ & 11 & 18 & 22 & 51 \\
$71-80$ & 31 & 40 & 11 & 82 \\
$81-90$ & 12 & 10 & 14 & 36 \\
$91-100$ & 4 & 1 & & 5 \\
\hline Total & 63 & 73 & 56 & 192 \\
\hline
\end{tabular}

Table 1: Distribution of pasta by age and classification. 


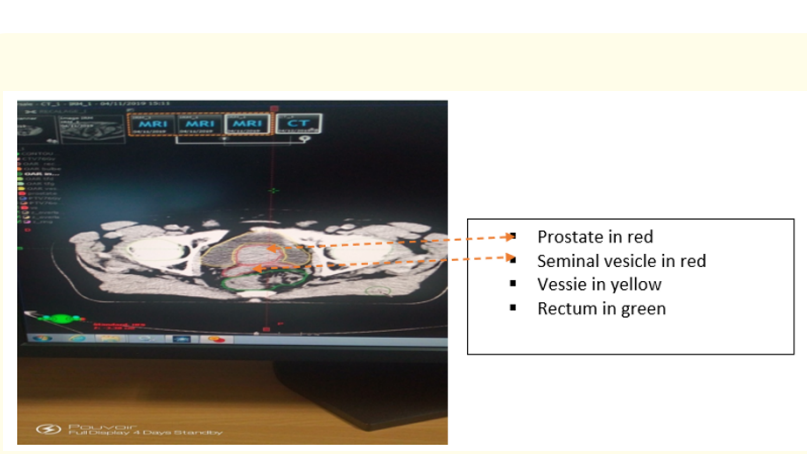

Figure 1: Contouring target volumes and organs at risk in axial cut.

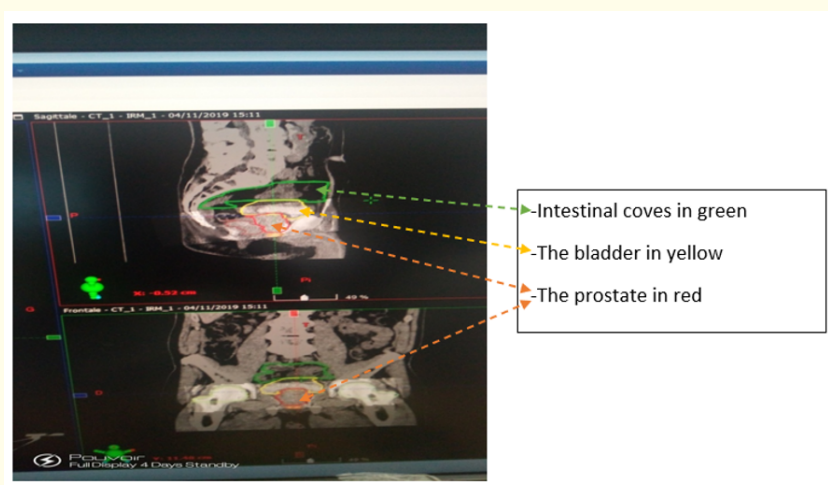

Figure 2: Contouring target volumes in sagittal and coronal cuts.
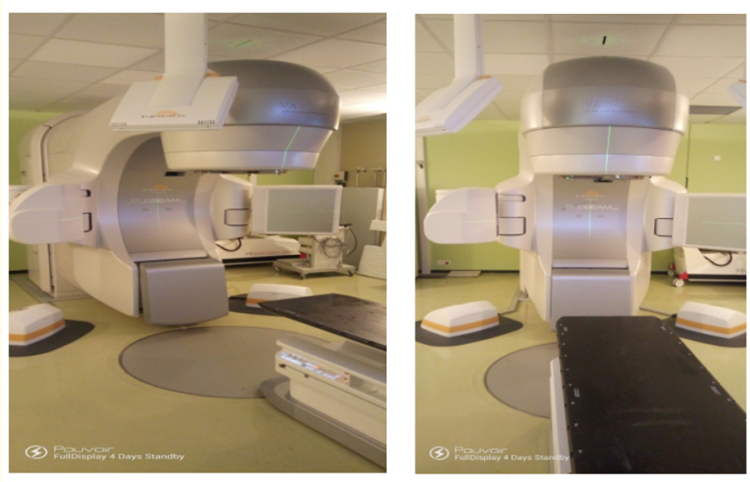

Figure 3: Novalis Tx device from Clarac Hospital of the Martinique University Hospital.

\begin{tabular}{|l|c|c|c|c|}
\hline $\begin{array}{c}\text { Post-radique } \\
\text { response in 12 } \\
\text { months }\end{array}$ & $\begin{array}{c}\text { Intermediate } \\
\text { Risk }\end{array}$ & $\begin{array}{c}\text { High } \\
\text { Risk }\end{array}$ & $\begin{array}{c}\text { Locally } \\
\text { Advanced } \\
\text { Risk }\end{array}$ & Total \\
\hline $\begin{array}{l}\text { Complete } \\
\text { remission }\end{array}$ & 60 & 69 & 46 & 175 \\
Progression & 3 & 2 & 7 & 12 \\
Lost sight & 1 & 1 & 2 \\
Death & 63 & 73 & 56 & 192 \\
\hline Total & & & 2 & 3 \\
\hline
\end{tabular}

Table 2: Post-radique therapeutic evaluation.

\section{Discussion}

Prostate cancer is the most common cause of male cancer and remains the leading cause of cancer death in men in Martinique. The improvement of diagnostic means and the development of high-performance technical trays now allow the University Hospital Centre of Martinique to continue its medical development. The Martinique Cancer Registry has been involved in epidemiological surveillance of cancers since 1981 by allowing the collection and analysis of incidence and mortality data. Radiation therapy in combination with hormone therapy remains the standard gold in the management of prostate cancer in localized stages especially for intermediate and high risks and then in locally advanced stages [3].

In our series of studies, the most representative age group was between71 and 80 years with an average age of 71 years. This average is close to that reported in the literature. The youngest patient was 54 years old and the oldest was 93 years old.

We used the D'Amico classification to classify patients and found that high-risk localized stage was the most represented group followed by intermediate risk. Also, we found that a significant number of patients in complete remission after an annual evaluation were from the high-risk localized group.

In our study, all patients had received are commended extension assessment depending on the stage of diagnosis including the thoraco-abdomino pelvien scanner with or without bone scans and/or a PET scan - Choline. 
PET-CT at 18-FDG has no indication in the lymph node balance because of low glucose metabolism in prostate cancer.

Thus, as part of the extension assessment, the recommendations currently use PET at 18 F-Choline (TEP-Choline) or PET at 68Ga-PSMA (TEP-PSMA).

In our series, patients had benefited from PET-Choline because it detects subclinical bone lesions early. In the absence of lymph node invasion( $\mathrm{pN} 0)$, radiotherapy was targeted only on the entire prostate and pelvis.

In the event of a node invasion $(\mathrm{pN})$, the regional lymph node areas were irradiated [4]. Intermediate-risk localized patients had received short-term hormone therapy combining Bicalutamide 50mg (Casodex)for 28 days and Triptorelin (Decapeptyl) $22.5 \mathrm{mg}$ in a single injection.

Patients at high-risk and locally advanced stages had alongterm hormone rapid combining Bicalutamide 50mg (Casodex) for 28 days and Triptorelin (Decapeptyl) $22.5 \mathrm{mg}$ injection every 6 mois. The annual post-radiation therapeutic evaluation in this study found a complete remission rate of 175 patients at all stages. This objective response after one year of treatment shows how this new technique improves the quality of life of patients benefiting from this scientific evolution. In addition, there was a progression in 12 patients and three deaths. It should be noted that these results are the result of technological advances in radiotherapy for the benefit of prostate cancer patients.

Thus the VMAT technique (Irradiation with Volumetric Intensity Modulation by ArcTherapy) offers the possibility of irradiating cancerous tissues with more precision than convent radiotherapy, thanks to a modulation of the irradiation beams on a complete arc of 360 degrees. It is a technique that therefore combines imageguided irradiation (IGRT) with the modulation of intensity [5]. Indeed, image-guided irradiation (IGRT) allows to control the correct position of the tumor target during treatment via ane acquisition of $3 \mathrm{D}$ volumetric image. It is justified by the anatomical and positioning variations occurring during irradiation. Intensity modulation, is a feature that allows to change during irradiation, the doses of rays depending on the volume to be irradiated. It is then possible to treat tumors of the xe-shaped form and to preserve the surrounding organs more, reducing acute toxicity and especially long-term toxicity [6-25].

\section{Conclusion}

The VMAT technique is one of the innovative radiotherapy techniques used in the priin charge of prostate cancer. The encouraging results found in this study suggest that the VMAT technique in the management of prostate cancer is part of the innovative therapeutic arsenal in radiation to improve patient outcomes, survival and quality of life.

\section{Bibliography}

1. Abdollah F., et al. "A competing-risks analysis of survival after alternative treatment modalities for prostate cancer patients: 1988-2006". European Urology 59.1 (2011): 88-95.

2. Albertsen PC., et al. "13-year outcomes following treatment for clinically localized prostate cancer in a population based cohort". Journal of Urology 177.3 (2007): 932-936.

3. Calais da Silva F., et al. "Locally advanced and metastatic prostate cancer treated with intermittent androgen monotherapy or maximal androgen blockade: results from a randomised phase 3 study by the South European Uroncological Group". European Urology 66 (2014): 232-239.

4. Salomon L., et al. "CCAFU Recommendations 2013: Prostatecancer”. Progrès en Urologie 23 (2013): 69-101.

5. Gandaglia G., et al. "Intensity-modulated radiation therapy leads to survival benefit only in patients with high-risk prostate cancer: A population-based study". Annals of Oncology 25.5 (2014): 979-986.

6. Anandadas CN., et al. "Quality of life in men treated for early prostate cancer: A prospective patient preference cohort study". Journal of Cancer Therapy 2.4 (2011): 448-457.

7. Ferrer M., et al. "Quality of life impact of treatments for localized prostate cancer: Cohort study with a 5 year follow-up". Radiotherapy Oncology 108.2 (2013): 306-313.

8. Gore JL., et al. "Correlates of bother following treatment for clinically localized prostate cancer". Journal of Urology 184.4 (2010): 1309-1315.

9. Hjälm-Eriksson M., et al. "Long-term health-related quality of life after curative treatment for prostate cancer: A regional crosssectional comparison of two standard treatment modalities". International Journal of Oncology 46.1 (2015): 381-388. 
10. Nieder AM., et al. "Radiation therapy for prostate cancer increases subsequent risk of bladder and rectal cancer: A population based cohort study". Journal of Urology 180.5 (2008): 2005-2010.

11. Pardo Y., et al. "Quality-of-life impact of primary treatments for localized prostate cancer in patients without hormonal treatment". Journal of Clinical Oncology 28.31 (2010): 4687-4696.

12. Petrelli F., et al. "Radical prostatectomy or radiotherapy in high-risk prostate cancer: A systematic review and metaanalysis". Clinical Genitourinary Cancer 12.4 (2014): 215-224.

13. Pickles T., et al. "Brachytherapy or conformal external radiotherapy for prostate cancer: A single-institution matched-pair analysis". International Journal of Radiation Oncology • Biology - Physics 76.1 (2010): 43-49.

14. Punnen S., et al. "Long-term health-related quality of life after primary treatment for localized prostate cancer: Results from the CaPSURE registry". European Urology 68.4 (2015): 600608.

15. Schapira MM., et al. "Effect of treatment on quality of life among men with clinically localized prostate cancer". Medical Care 39.3 (2001): 243-253.

16. Schymura MJ., et al. "Factors associated with initial treatment and survival for clinically localized prostate cancer: Results from the CDC-NPCR Patterns of Care Study (PoC1)". BMC Cancer 10 (2010): 152.

17. Shinohara N., et al. "Longitudinal comparison of quality of life after real-time tumor-tracking intensity-modulated radiation therapy and radical prostatectomy in patients with localized prostate cancer". Journal of Radiation Research 54.6 (2013): 1095-1101.

18. Jones CU., et al. "Radiotherapy and short-term androgen deprivation for localized prostate cancer". The New England Journal of Medicine 365 (2011): 107-118.

19. Fizazi K., et al. "Androgen deprivation therapy plus docetaxel and estramustine versus androgen deprivation therapy alone for high-risk localised prostate cancer (GETUG 12): a phase 3 randomised controlled trial". Lancet Oncology 16 (2015): 787794.
20. Siddiqui KM., et al. "Comparative morbidity of ablative energy-based salvage treatments for radio-recurrent prostate cancer". Canadian Urological Association journal = Journal de l'Association des urologues du Canada 9 (2015): 325-329.

21. Crawford ED TB., et al. "A phase III extension trial with a 1-arm crossover from leuprolide to degarelix: comparison of gonadotropin-releasing hormone agonist and antagonist effect on prostate cancer". Journal of Urology 186 (2011): 889-897.

22. Group PCTC. "Maximum androgen blockade in advanced prostate cancer: an overview of the randomised trials". Lancet 355 (2000): 1491-1498.

23. Nair B., et al. "Early versus deferred androgen suppression in the treatment of advanced prostatic cancer". Cochrane Database of Systematic Reviews (2002): CD003506.

24. Calais da Silva FE., et al. "Intermittent androgen deprivation for locally advanced and metastatic prostate cancer: results from a randomised phase 3 study of the South European Uroncological Group". European Urology 55 (2009): 1269-1277.

25. Salonen AJ., et al. "The FinnProstate Study VII: intermittent versus continuous androgen deprivation in patients with advanced prostate cancer". Journal of Urology 187 (2012): 20742081.

\section{Volume 5 Issue 7 July 2021}

(C) All rights are reserved by Nkoua Epala B., et al. 